\title{
El modelo de la complejidad frente a los modelos psicológicos tradicionales de la ansiedad ante la muerte
}

\author{
LeONARDo Yovany Álvarez ${ }^{1}$
}

\section{Resumen}

El tema de la ansiedad ante la muerte ha sido objeto de investigación desde diferentes perspectivas psicológicas, hace parte de muchos cursos de tanatología para estudiantes del área de la salud y, también, de algunos cursos para operadores encargados de trabajar con pacientes terminales. La ansiedad ante la muerte es un fenómeno complejo que involucra las capacidades de afrontamiento del individuo pero a su vez implica la confrontación de la experiencia vital, la integración de la misma, la resolución de expectativas, el duelo, en un momento que se torna implacable. En la presente revisión sobre la ansiedad ante la muerte, se analizan algunos de los modelos psicológicos individualistas, vinculares y psicosociales. Se discuten los alcances y limitaciones de la perspectiva explicativa unidireccional de la ansiedad ante la muerte desde la cual se la aprecia como un evento generador de defensa psicológica y de reacciones de evitación, ya sea mediante la postergación, la atribución externa, la búsqueda de la plenitud en la individuación dejando de lado las contradicciones, ambigüedades, borrosidades y ambivalencias que dicho fenómeno psicológico tiene, lo cual deriva variadas implicaciones para su comprensión e intervención no sólo dentro de la institución sanitaria sino desde el ámbito social y comunitario.

Palabras clave: ansiedad, muerte, complejidad, vincular, modelos psicológicos.

\section{Title}

The model of complexity against common psychological anxiety towards death models

\begin{abstract}
Anxiety towards death has been a subject of investigation since different psychological perspectives. It takes part in tanatology courses directed for health careers students and for health professional attending terminal patients. Anxiety towards death is a complex phenomenon which involves the individual coping skills but also the confrontation of the
\end{abstract}

1 Docente, Programa de Psicología, Universidad Autónoma de Bucaramanga, Bucaramanga, Colombia. Recibido: 10-02-2010

Revisado: 02-02-2010

Aceptado: 14-04-2010

Álvarez L. Y., El modelo de la complejidad frente a los modelos psicológicos tradicionales de la ansiedad ante la muerte 
individual's whole life experience, the integration of it and the expectations and living resolutions, the mourning into a implacable time. This checking is about anxiety towards death psychological models, such as: Psychosocial, individualists and bonding ones. Linear explanations, limits and scopes on anxiety towards death are retaken, especially when it is seen like a psychological defense, avoiding reaction,deferrings, external attribution, individualistic plenty seeking, leaving away the contradictions, ambiguities, blurrings this phenomenon has and the implications to understand and intervene it.

Key words: anxiety, death, complexity, bond, psychological models.

\section{Introducción}

Las nuevas investigaciones sobre psicopatología y otros fenómenos psicológicos (depresión, ira, psicosis) se vienen orientando desde una perspectiva compleja[1]. La ansiedad ante la muerte exhibe propiedades como la borrosidad, la ambigüedad y la contradicción, que se ajustan a un marco de lectura complejo, capaz de considerar al sujeto con sus ambivalencias, incertidumbres e insuficiencias. Tenemos identidad porque nunca somos los mismos, y sentimos plenitud al individuarnos y miedo de separarnos de los demás. La falta de coherencia y de consistencia es constitutiva de la identidad personal (self), desde la cual experimentamos la muerte. La identidad personal es por definición inestable: la plenitud coexiste con la ansiedad, dado que dicha identidad es inconsistente[2].
Las teorías psicológicas sobre la ansiedad ante la muerte se han construido en torno a variadas unidades de análisis: "el sí mismo", "la individuación"[3], las actitudes, la emoción (ansiedad ante la muerte), los modelos para afrontar el "ser o no ser"[4] o la plenitud vital[5], entre otras. Unas lecturas han estado centradas en lo individual, otras en lo psicosocial y otras en lo vincular.

Desde una perspectiva existencialista (individual), se plantea el grado de plenitud vital[6] como indicador de alta o baja ansiedad ante la muerte. Sin embargo, una posición contraria a la anterior sostiene que en la negación de la satisfacción de la vida se hallaría una menor ansiedad ante ésta -la actitud existencial de aceptación, no desiderativa[7].

En el caso de la perspectiva vincular, se plantea el grado de individuación como el indicador de la mayor o menor ansiedad experimentada hacia la muerte; esto es, el grado en que una persona ha desarrollado autonomía personal para guiarse de manera autodeterminada en su vida, sin temores de ser ella misma, aun en franca oposición al grupo, la sociedad y la familia, experimentándose como un ser separado, lo cual la llevaría a sentir menor ansiedad ante la muerte. Por otra parte, en oposición a ello pero desde la misma perspectiva vincular, 
está el planteamiento opuesto, es decir, que ese individuo "separado" no sería quien experimentaría menor ansiedad, sino por el contrario, mayor ansiedad ante la muerte.

Sin embargo, la lectura que se ha dado al fenómeno de la ansiedad ante la muerte, desde lo vincular, ha sido lineal en extremo, sobre todo, cuando dicha experiencia tiene un comportamiento paradójico, es decir, en ella coexisten la plenitud de la separación y el temor concomitante de dejar de ser con el grupo, con la sociedad, con la familia y con el mundo.

Esta aproximación admite que el límite para estos sentimientos es borroso, puesto que se experimentan al mismo tiempo uno y otro, y pueden ser leídos teóricamente desde la lógica de lo ambivalente, de lo contradictorio. La perspectiva de las actitudes, por ejemplo, parte de un supuesto de linealidad en el cual los sujetos buscan un orden, una coherencia y una congruencia entre sus creencias, sentimientos y actos; esto, aplicado al fenómeno de la ansiedad ante la muerte, propone una linealidad en el cual la contradicción de la que se habló con precedencia sería un imposible.

Desde el punto de vista de Durlak, Horn y Kass[8], es teóricamente posible que las actitudes sean multidimensionales o complejas, es decir, que se experimenten actitudes múltiples fren- te al mismo asunto, para el caso, hacia la muerte. Esto significa que una persona podría tener sentimientos positivos y negativos al mismo tiempo respecto a la misma.

Una revisión de algunos de los modelos psicológicos sobre la ansiedad ante la muerte, deja claro que la concepción unidimensional parece ser la que se destaca en la investigación[911] y que el paradigma de la complejidad que ha penetrado en la psicología social desde hace algún tiempo[12] no parece haber sido tenido en cuenta como una alternativa posible para comprenderla de una manera más amplia, desde lo caótico y contradictorio, donde la muerte sería una realidad no accesible desde un marco simplificador, no lineal y constrictiva.

Diferentes investigadores han reconocido que el paradigma de la complejidad tiene aplicación en las ciencias de la vida y en las relaciones complejas entre componentes, según lo expone Capra[13]. En este sentido, se propone que la ansiedad ante la muerte puede abordarse como un fenómeno complejo, al cual le resultan aplicables las propiedades del caos, la emergencia y autoorganización, entre otras, como se comentará más adelante.

Los modelos centrados en la autoestima como unidad explicati$\mathrm{va}$ [14] de la ansiedad ante la muerte, hacen énfasis en un "sí mismo" que 
evoluciona hacia la integración de la experiencia vivida, en la que su aceptación proporciona una sensación de plenitud que permite asumirla. Según este modelo, el aprecio por sí mismo cedería progresivamente su espacio al desprendimiento, de tal manera que el "sí mismo" sería desinteresado, en el cual la ansiedad hacia la muerte sería baja.

Entre los modelos de autoestima, el de construcción biográfica[15] sigue una línea similar al anterior, pero tiene una naturaleza basada en el lenguaje, es decir, los individuos construirían relatos sobre la propia vida contados por sí mismos como intérpretes de la misma. En dicho relato, la identidad personal se iría integrando progresivamente, de lo cual resultaría una percepción más completa y diversa de la experiencia de vida, lo que derivaría en una disminución de la ansiedad ante la muerte.

En una línea teórica diferente, pero convergente desde un ángulo más psicosocial, se plantea un modelo en una sucesión de fases en las cuales la persona va progresivamente avanzando hacia una mayor integración, con la cual disminuyen sus temores ante la muerte, en particular, en la fase de integridad $v s$. desesperanza: la persona ve su vida como un todo significativo y resuelve su crisis ante la muerte de una manera favorable, sin temores intimidatorios[16].
En este punto viene la similitud con los planteamientos teóricos de las perspectivas de la autoestima, ya que la integración del ego sería el antecedente de una fase de generación en oposición a una de estancamiento. En esta fase, la conciencia de mortalidad haría emerger la ansiedad por la muerte[17], pero el "sí mismo" se expandiría, incluyendo otros elementos en el mismo sistema social y cultural.

La unidimensionalidad que siguen estos planteamientos es clara, en tanto plantean una serie de estados de expansión del yo en los cuales el individuo supera una serie de barreras emocionales. La integración vendría como un proceso secuencial conseguido por la superación de la crisis en el desarrollo de la autoconciencia.

Sin embargo, la idea de "no retorno", sino de superación de estados a los cuales no se vuelve, deja fuera una comprensión en espiral de la experiencia de la ansiedad ante la muerte y de la explicación misma del caos y la crisis en los procesos de cambio, pues tenemos identidad porque nunca somos los mismos; esto significa que el "sí mismo" es paradójico en su forma de funcionar, de organizarse, y en dicha organización sigue leyes de caos, de retorno y avance, de ser y no ser, lo cual introduce una comprensión diferente de los procesos de individuaciónseparación, y al mismo tiempo, del ser y no ser, de la identidad, del sí mismo 
y de la autoestima. Ésta se plantea como una coexistencia de confianzadesconfianza, consistencia-inconsistencia y estabilidad-labilidad, que le serían propias.

Las teorías de control del terror establecen como unidad de análisis "la ilusión de invulnerabilidad", la cual funcionaría como una defensa frente a la ansiedad ante la muerte; esto es, las personas se protegerían de la ansiedad ante la muerte mediante creencias acerca de su presentación futura, no en el presente[18]. Dicha creencia tendría una función adaptativa de evitación que retiraría de la vida de los individuos la perspectiva de la muerte en el momento actual. Al pensar en la muerte como un evento del futuro, la ilusión de invulnerabilidad aumenta, reforzando la propia seguridad y bienestar[19]. Las personas con mayor ilusión de invulnerabilidad planearían su vida más en el futuro[20] y aquellas con menor ilusión de invulnerabilidad, lo harían más en el presente[21].

Diferentes estudios se han aproximado al tema y han hallado que existen mayores niveles de ansiedad hacia la muerte en los más jóvenes que en los de mayor edad[22]; otros han encontrado resultados diferentes. Por ejemplo, algunos autores[23] apoyan la hipótesis de que parece existir una relación inversa entre la edad y el miedo hacia la muerte; en particular, encontraron estas relaciones inversas en estudiantes, quienes hasta los treinta años reportaron mayor miedo a la muerte que los adultos entre 40 y 69 años.

En poblaciones de ancianos y jóvenes, un estudio español[24] encontró confirmación para la relación inversa entre edad y miedo a la muerte. Estos investigadores encontraron que, en los ancianos y en los hombres, la ansiedad ante la muerte fue menor que en los jóvenes. Estos hallazgos fueron confirmados por otros investigadores[25] quienes, a su vez, hallaron que los niveles de ansiedad o depresión hacen que se incremente la ansiedad ante la muerte, independientemente de la edad o el sexo. Entre otras explicaciones, una de las más notorias es que los adultos jóvenes tienden a iniciar sus profesiones y la construcción de un proyecto familiar que impide aceptar la muerte con la misma perspectiva de inevitabilidad que lo harían las personas adultas mayores.

El modelo "actitud hacia la muerte" ha sido explorado sin hallazgos concluyentes respecto a la edad; al parecer, los ancianos tienden a reportar una actitud de aceptación mayor que los jóvenes hacia la muerte y aunque esto no quiere decir que estén de acuerdo con morir, los jóvenes se hallan más influenciados por alcanzar la conclusión de una vida feliz[26]. Esta propuesta introduce explicaciones que relativizan la experien- 
cia de ansiedad en función de la cercanía o lejanía del estímulo que causa aversión (en este caso, la muerte), pero no explican la manera como esta defensa se articula con otros factores psicosociales más allá de la sola función adaptativa; tampoco muestra de qué manera la ilusión de invulnerabilidad coexiste al lado de la misma vulnerabilidad y el individuo alterna la vulnerabilidad-invulnerabilidad en un proceso en el cual habría mayor compromiso con el presente $y$, en otras ocasiones, con el futuro en una inestabilidad paradójica no unidimensional.

La teoría de control del terror[27] sostiene que las creencias de inmortalidad e invulnerabilidad con las cuales se socializa a un individuo, actuarían como un escudo simbólico de protección. Las teorías de control del terror también asocian la autoestima integrada, libre de individualismos, incluyente, como un elemento fundamental en la baja ansiedad ante la muerte[28]. Las culturas promoverían procesos de socialización en los cuales predominarían valores de autosuficiencia, omnipotencia, invulnerabilidad y compleción, dirigidos a la evitación de la muerte[29]. Por su parte, la vanidad de concebirse diferente a los demás, el no compartir con los otros la fragilidad, sería una manifestación más de la ansiedad ante la muerte.

También en los estilos de vida protectores de la salud, por ejemplo, ha- bría una conducta defensiva frente a la muerte: la vida vivida con intensidad sería una forma defensiva frente a la muerte. Los elementos de ambigüedad que dejan de lado la propuesta están relacionados con la contraparte simultánea de impotencia, insuficiencia y falta de compleción, que se oponen a esas tendencias. Las carencias y sus correspondientes excesos y matices ante la experiencia de la muerte, hacen parte de la realidad de la socialización. Se socializa para ser fuerte ante lo adverso, para superar la dificultad teniendo en cuenta que existe la flaqueza, la debilidad como parte de ese mismo proceso de defensa reafirmadora de la vida, el pesimismo al lado del optimismo, la muerte al lado del deseo de sobrevivir.

Un tanto diferentes son los planteamientos de las teorías de las ilusiones positivas, pues la unidad de análisis es el locus de control, esto es, la atribución causal que hacen las personas de la muerte. De acuerdo con varios investigadores[30], lo que predeciría la ansiedad ante la muerte en los individuos sería la creencia de causalidad que adjudican a este hecho: las personas con un alto locus de control interno tenderían a mostrar una mayor ansiedad ante la muerte que las personas con locus de control externo. Esto se debe a que los individuos con locus de control externo atribuyen la muerte a cosas del destino, a razones que no están bajo su control y, por lo tan- 
to, tienen una perspectiva más relajada de la misma, en tanto que las personas con un locus de control interno tienden a pensar que la muerte está más bajo su control y, por lo tanto, le temen más.

$\mathrm{Al}$ explorar las posibles relaciones con la personalidad, ésta mostró ser una variable estrechamente correlacionada con la ansiedad o la preocupación por la muerte[31]. La variable locus de control ha revelado una relación significativa entre la actitud hacia la muerte y el locus de control: cuando éste es interno, se genera menos ansiedad sobre la muerte que cuando es externo[32]. Aquí aparece la versión clara acerca de la falta de complementariedad, si no de la disyuntiva, con respecto a la posibilidad de experimentar sentimientos ante la experiencia de la muerte.

El locus de control es interno o externo (disyuntivo), pero no hay posibilidad de coexistencia. En el primer caso, el individuo se atribuye una gran responsabilidad con respecto a su destino y a sí mismo por razones que aparentemente él puede controlar, y se experimenta una gran ansiedad ante la muerte. En el segundo, no asume dicha responsabilidad, sino que la atribuye a factores externos. No obstante, no se plantea como posible que estas dos posiciones se alternen. El modelo es así: simplificador y unidi- reccional. Tanto la posibilidad de controlar el no morir como la de no controlarlo, hacen parte de la vida de los individuos, sin descartar, por ejemplo, que haya algún tipo de predominancia en algunas circunstancias vitales.

Desde una perspectiva cognitiva, la teoría de los modelos personales[33] propone que la ansiedad ante la muerte procede de la falta de sentido que ésta tiene dentro del sistema de modelos. Aquí, la autoestima tiene centralidad puesto que la teoría la considera incluida en los mismos polos de una muestra de modelos personales[34]. En este caso, la oposición con una perspectiva compleja y paradójica es muy clara, dado que el autor propone un modelo como bipolar con opuestos categóricos totalmente excluyentes, pero nunca coexistentes: los estados emocionales intermedios entre ansiedad y aceptación de ella no serían posibles. Se experimentaría una en la medida en que se aleja de la otra.

Los planteamientos de Rogers[35] mantienen en una línea muy similar a la de la autoestima, que la muerte es una negación total de la experiencia del sí mismo, la cual deviene cuando es confrontada con el sentimiento de valía personal dado que dicha percepción de incongruencia es incompatible con el self. Este hecho psicológico generaría un alto grado de ansiedad. De aquí se deduce que una mayor 
aceptación de la muerte sobrevendría, si la necesidad de autoconsideración positiva no fuera tan acentuada.

Por otra parte, en el marco del sentimiento de autoactualización, estas defensas no serían necesarias y corresponderían a la persona "autoactualizada", en la cual existe un funcionamiento óptimo, la apertura completa a la experiencia en un grado tal, inclusive, que pudiera llegar a ser una experiencia interesante, más que amenazante[36]. En conclusión, podría decirse que el nivel de autorrealización está relacionado de manera inversa con una mayor o menor ansiedad ante la muerte. Aquí se tiene que la autorrealización sería un estado de llegada, un punto en una secuencia de estados previos y, una vez más, una fase de estabilidad, de consistencia y de equilibrio, en la cual la posibilidad de lo caótico, ambiguo, desequilibrado e inconsistente coexista con el equilibrio en esa autoactualización.

Desde una aproximación vincular[37], sugiere que la individuación está relacionada con el miedo a la muerte, toda vez que si la persona no se logra concebir separada del grupo, esto podría llevarla a experimentar ansiedad ante la muerte, por temor a la soledad. Maslow[38] sostiene, en contraposición, que cuando la persona alcanza la separación del grupo, su experiencia de plenitud sería alta y, por tanto, su miedo a la muerte bajó. De acuerdo con este autor, si la persona se mantiene en los grupos no está expuesta a afrontar la diferenciación que le expone a percatarse de su debilidad, de su vulnerabilidad y de sus carencias, con las posibilidades de extinción que ello conlleva. La aceptación de la norma social sería así una manera de disminuir la ansiedad ante la muerte, en la medida en que se establece una causa común con los demás[18].

Esta perspectiva introduce la posibilidad de la plenitud de la individuación, pero no deja claro que simultáneamente pueda darse el miedo a dejar el grupo y que en esta paradoja se pueda experimentar una ansiedad y una plenitud de manera simultánea y, por tanto, la ansiedad ante la muerte no resulte ser una experiencia tan polar ni unidimensional.

La perspectiva compleja se está introduciendo en la investigación psicológica[40] y la ansiedad ante la muerte es un tópico en el cual resulta pertinente aplicarla. Desde una perspectiva compleja, la linealidad no sería el marco de lectura de la experiencia de la ansiedad ante la muerte, so pena de resultar demasiado simplificadora. $\mathrm{La}$ ansiedad surge del sentimiento de separación y se une al sentimiento concomitante de plenitud al alcanzarla. Desde este punto de vista, la ansiedad ante la muerte es un fenómeno caótico, confuso, que muestra que puede 
ser un sistema complejo con una fluctuación sujeta a la ambigüedad que impide tener certezas sobre el fenómeno mismo.

La borrosidad plantea que la posibilidad de hallar plenitud frente a la muerte y al mismo tiempo ansiedad, es amplia, sin fronteras entre un sentimiento y el otro[41], sin abismos infranqueables, haciendo la aplicación de lo planteado por Munné[42]. La ansiedad ante la muerte seguiría una lógica ambigua, en medio del paralelismo plenitud-ansiedad (principio de paralelismo). Las posibilidades de experiencias ansiosas ante la muerte no seguirían una lógica categórica[43] para los sistemas complejos en general. La ansiedad ante la muerte sería una experiencia inestable, caótica, conflictiva, incierta, no formulada desde algo que evitar sino desde la interacción creativa de la plenitud y el miedo. Es necesario, así, extraer de la lógica lineal unidimensional y simplificadora el tema, extraerlo de la mirada ordenada, predecible y unidimensional, para inscribirla en lo ambiguo, en lo complementario (miedo-plenitud) en una lógica circular.

\section{Bibliografía}

1. Munné F. El retorno de la complejidad y la nueva imagen del ser humano: hacia una psicología compleja. Revista Interamericana de Psicología. 2004; 38:23-9.

2. Munné F. El self paradójico: identidad como sustrato del self. En: Caballero
D, Méndez M, Pastor J., compiladores. La mirada psicosociológica. Grupos, procesos, lenguajes y culturas. Madrid, España: Biblioteca Nueva. 2000;74050.

3. Rogers CR. A way of being. Boston: Houghton-Mifflin. 1980.

4. Kelly GA. The Psychology of personal constructs. Nueva York: Norton; 1955.

5. Maslow AH. Toward a psychology of being. 2a. edición. Nueva York: Van Nostrand Reinhold. 1968;72-91.

6. Erickson EH. The life cycle completed. New York: Norton. 1982.

7. Breznitz S. Cry wolf: the psychology of false alarms. New Jersey: Lawrence Erlbaum; 1983.

8. Durlak JA, Kass RA. Clarifying the measurement of death attitudes. Omega. 1981-1982;12:129-41.

9. Marshall VW. Death and dying. In: Man Gen D, Peterson W, compilators. Research instruments in social gerontology. Clinical and social psychology. Minneapolis: University of Minnesota Press. 1982;303-81.

10. Vargo ME, Batsel WM. Relationship between death anxiety and components of the self actualization process. Psychological Reports. 1981;48:8990.

11. De Paola M, Neimeyer R, Lupfer K, Fiedler N. Research about the anxiety of death in diferent population. Arkansas: Ohio Press. 1992;12-35.

12. Munné F. Las teorías de la complejidad y sus implicaciones en las ciencias del comportamiento. Revista Interamericana de Psicología. 1995;29:1-12.

13. Capra F. Las conexiones ocultas. Barcelona: Anagrama. 2003.

14. Dickstein LD. Attitudes toward death anxiety and social desirability. Omega. 1977-1978;8:369-78. 
15. Marshall VW. Death and dying. In: Man Gen D, Peterson W, compilators. Research instruments in social gerontology. Clinical and social psychology. Minneapolis: University of Minnesota Press. 1982;303-64.

16. Erickson EH, Erickson JM, Kiunick HQ. Vital involvement in old age. Nueva York: Norton. 1986.

17. Neugarten B. Personality and aging. In: Birren JE, Schaie KW, compilators. Handbook of the psychology of aging. New York: Van Nostrand Reinhold. 1984;625-49.

18. Becker E. The denial of death. New York: Free Press. 1973.

19. Breznitz S. Cry wolf: the psychology of false alarms. New Jersey: Lawrence Erlbaum. 1983.

20. Neimeyer RA, Bagley KL, Moore MK. Cognitive structure and death anxiety. Death Studies. 1986;10:270-90.

21. Vargo ME, Batsel WM. Relationship between death anxiety and components of the self actualization process. Psychological Reports. 1981;48:8996.

22. James F, Lloyd G, Groff T. On death and dying. New York: Mac Millan. 1999.

23. De Paola M, Neimeyer R, Lupfer K, Fiedler N. Research about the anxiety of death in different population. Arkansas: Ohio Press. 1992;12-35.

24. Martínez T, Valera R, Frances D. Toward a composition of death features. Washington: SRDR Press. 2001;15-56.

25. Keller JW, Sherry D, Piotrowski C. Perspective on death: a developmental study. Journal of Psychology. 1984;116:137-42.
26. Young R, Cullen G. Cognitive structure and death process in ages. New York: PSES Press. 1996;23-54.

27. Rosenblatt A, Greenberg J, Solomon S, Pyszczynski T, Lion D. Evidence for terror management theory: the effect of mortality salience on reactions to those who violate or uphold cultural valves. Journal of Personality and Social Psychology. 1989;57:681-90.

28. Davis SF, Martin DA, Wilee CT, Voorhees JW. Relationship of fear of death and level of self-esteem in college students. Psychological Reports. 1978;42:419-22.

29. Firestone RW. The fantasy bond: the structure of psychological defenses. Nueva York: Human Sciences Press. 1985.

30. Vargo ME, Black F. Fear of death, approaches to this aspect. New York: Camalav Science Press. 1984.

31. Kuperman SK, Goleen SJ. Personality correlates of attitude toward death. Journal of Clinical Psychology. 1978;34:661-3.

32. Hyams F, Domino R, Spencer R. Dynamics and development the approaches towards death and anxiety. Washington: University Press. 1987.

33. Kelly GA. The psychology of personal constructs. Nueva York: Norton. 1955.

34. Neimeyer RA, Bagley KL, Moore MK. Cognitive structure and death anxiety. Death Studies. 1986;10:270-92.

35. Rogers CR. A way of being. Boston: Houghton-Mifflin. 1980;76-89.

36. Rogers, C. R. A way of being. Boston: Houghton-Mifflin. 1980;64-91.

37. Rank O. Beyond psychology. New York: Dover. 1958. 
38. Maslow AH. Toward a psychology of being. $2^{\text {nd }}$ edition. Nueva York: Van Nostrand Reinhold. 1968;65-88.

39. Scott J. Approaches toward complexity in reality. Washington: Narvingham. 1991.

40. Zadeh J. Complexity and the logos in reality interpretation. Washington: Kourtdefron. 1987.
41. Munné F. ¿Qué es la complejidad? En: Munné F, coordinador. Encuentros en psicología social y de las organizaciones. Málaga: Aljibe. 2005;7-9.

42. Kosko B. Pensamiento borroso. La nueva ciencia de la lógica borrosa. Barcelona: Crítica. 1993.. 